\title{
Oral Lichen Planus
}

National Cancer Institute

\section{Source}

National Cancer Institute. Oral Lichen Planus. NCI Thesaurus. Code C7406.

A chronic, autoimmune inflammatory condition of the mucous membranes in the oral cavity that affects approximately two percent of the population and is most often seen in middle aged women. It is characterized by white, lacy patches; red, swollen tissue; papules and plaques; or open sores. The lesions are typically bilateral. 